\title{
Die illyristische Versuchung
}

\author{
REINHARD LAUER \\ (Göttingen)
}

\section{Einführende Überlegungen}

Der Illyrismus stellt aus heutiger Sicht eine kroatische Kulturbewegung in der ersten Hälfte des 19. Jahrhunderts dar, die zugleich die Stilformation der Romantik vertritt Darüber hinaus hat er in wesentlichem Maße zur Festigung des modernen kroatischen Nationalbewusstseins beigetragen und die Weichen für die Schaffung einer einheitlichen kroatischen Literatursprache gestellt. Dass der Illyrismus gleichzeitig auch Argumente für eine kulturelle und politische Einheit aller Südslawen geliefert hat und im "Jugoslawismus“ des Bischofs Štrosmajer seine Fortsetzung fand, gehört zu den Widersprüchen, oder besser: zu der prinzipiellen Offenheit, mit der im 19. Jahrhundert politisch und kulturpolitisch gedacht wurde. Trotz allem: wie sehr sich die illyrische Bewegung auch als eine all-südslawische verstand, also außer den kroatischen Stämmen auch Slowenen, Serben, Montenegriner, Bosnier und Bulgaren in ihre Bestrebungen einbeziehen wollte - sie blieb, historisch betrachtet, ein kroatisches Ereignis. Keine der angesprochenen Nationen, außer den Kroaten, folgte dem illyristischen Sog; nur ganz wenige Literaten machten sich die illyristische Sache so zu eigen, wie sie Ljudevit Gaj, der Wortführer des Illyrismus, in seinen Aufrufen (Oglasi) verfocht. Zu nennen wären die Serben Teodor Pavlović (für nur kurze Zeit) und Sava Tekelija, kein Montenegriner, kein Bulgare. Die große, fast alleinige Ausnahme bildet der aus der Steiermark gebürtige Slowene Stanko Vraz, nach France Prešeren der beste slowenische Dichter seiner Zeit. Vraz verlässt nicht nur seine Dežela, sein steirisches Heimatland, um sich für dauernd in Zagreb niederzulassen, sondern gibt auch seine Sprache, den südoststeirischen Dialekt des Slowenischen, auf und schreibt bis zu seinem Tode im Jahre 1851 den größten Teil seiner Werke in der ,illyrischen“ Sprache, das heißt in der von Gaj, Brlić u.a. geformten kroatischen Literatursprache. Vraz ist, nebenbei bemerkt, der produktivste Autor im Kreise der Literaten des Illyrismus, von weiter literarischer Bildung und von unermüdlichem Unternehmungsgeist. Er ist ein Literat im vollen Wortsinn: Dichter, Übersetzer, Volksliedsammler, Literaturkritiker, Publizist, Zeitschriftengründer und Redakteur. Eine solche Gestalt wie Stanko Vraz ist es denn auch insonderheit, die die Frage nach der illyristischen Versuchung überhaupt aufkommen lässt: Wie 
konnte sich dieser hochbegabte Dichter aus seiner slowenischen sprachlichen Verankerung reißen, um an fremdem - illyristischem - Gestade eine neue literarische Heimat zu finden?

Die Analyse der Causa Stanko Vraz samt ihrer sprach-, literatur- und kulturhistorischen Kontexte, vermag uns, so scheint mir, die einmalige, im höchsten Maße unübersichtliche und vieldeutige Situation zu vergegenwärtigen, in der die neue slowenische ebenso wie die neue kroatische Literatur entstanden. Selbstverständlich geht es mir nicht darum, Prešeren und Vraz als zwei Kontrahenten in einem imaginären Duell vorzufuihren - obwohl etwa Antun Barac unumwunden Prešeren als den Hauptgegner (glavni Vrazov protivnik) ${ }^{1}$ von Stanko Vraz bezeichnet; vielmehr soll hier die Offenheit und Optionsfreiheit aufgezeigt werden, welcher die südslawischen Dichter in den Jahren zwischen 1830 und 1843 ausgesetzt waren. Sich in dieser verwickelten Situation zurechtzufinden und zu behaupten, erforderte mehr als nur nationalen, sprachlichen, kulturellen Instinkt; es hing ebensosehr von den Visionen in einem Felde $a b$, das in keinem Sinne bereits abgesteckt war. Lassen Sie mich, anstelle einer nochmaligen Nacherzählung der damaligen Ereignisse und Entscheidungen - das ist von Branko Drechsler, Slavko Ježić, Fran Petré, Antun Barac u.a. ${ }^{2}$ längst geleistet worden - lassen Sie mich also in aller Kürze einige modellartige Vorstellungen entwerfen - von der Idee des Illyrismus bzw. dem illyrischen Ideologem, wie ich es genannt habe, von den epistemologischen und ideengeschichtlichen Voraussetzungen, von der Sprachsituation in den südslawischen Ländern wie auch von den literarischen Strömungen, um von daher die Wahlmöglichkeiten, die Optionen slowenischer und kroatischer Schriftsteller zu rekonstruieren.

\section{Das illyrische Ideologem}

Zunächst möchte ich noch einmal ins Gedächtnis zurückrufen, was es mit der illyrischen Idee, besser: dem illyrischen Ideologem, auf sich hat. Ich habe mich vor Jahren mit diesem Gegenstand beschäftigt ${ }^{3}$ und kann hier knapp den grundlegenden Sinn und die wichtigsten Etappen in der Genese des illyrischen Ideologems nachzeichnen. Dieses ideologische Konstrukt wurde im Zeitalter des Humanismus entwickelt, um die auf der Balkanhalbinsel lebenden Südslawen, analog zu Italienern, Franzosen und Deutschen, ahnsehnliche antike Vorfahren zu bescheren. Mit einem Wort: Die gesamte nicht-griechische und nicht-römische Bevölkerung des Balkanraumes wurde zu Illyrern erklärt und diese als Vorfahren der Slawen reklamiert. Man gewann damit eine große Zahl illustrer Vorfahren - darunter Philipp von Mazedonien, Alexander der Große,

1 Barac 1964, Bd. I, S. 224.

2 Drechsler 1909; Ježić 1934; Petré 1939; Barac 1964 u. a.

3 Lauer 1974. 
Aristoteles, Kaiser, Päpste und Heilige. Da Dalmatien - später Krapina - als die Wiege des Slawentums angesehen wurde, haben hier auch die Stammväter der Slawen Čech, Lech und Rus/Mech ihre Heimat. Von Vinko Pribojević (Vincentius Priboevius), einem Dominikanerpater in Hvar, wurde erstmals, wie es Alois Schmaus ausgedrückt hat, der „Raumbegriff Illyrien mit slavischem Inhalt gefuillt" ${ }^{4}$ Pribojević konnte sich dabei auf ähnliche Überlegungen und Konstruktionen des Humanisten Juraj Šižgorić (Sisgoreus) aus Šibenik oder des Krakauer Geistlichen Maciej z Miechowa (Miechovitus) stützen. Die tatsächliche Wanderbewegung der Slawen und Avaren im frühen Mittelalter, das historische Schicksal der Thraker, der eigentlichen Illyrer oder der Dalmaten, überhaupt die Vorgänge der frühmittelalterlichen Geschichte in Südosteuropa spielten in diesem Geschichtsbild eine geringe Rolle. Vorrangig ging es um die Antikisierung der südslawischen Völker als „Illyriae gentes“, unter denen, etwa bei Pribojević, die Namen: Ruscia, Cassubia, Pruscia, Masovia, Moscovia, Polonia, Slesia, Moravia, Bohemia, Croatia, Bosna, Rascia, Servia und Bulgaria erscheinen. Die slowenischen Regionen sind hier übrigens noch ausgeblendet. Völker- bzw. Regionalkataloge werden in der Folgezeit bis hin zum Illyrismus als konstitutiver Bestandteil des illyrischen Ideologems immer wieder beschworen, in ragusanischen Wappenbüchern, bei Mauro Orbini, in der Stemmatographia von Pavao Ritter-Vitezović. Križanić subsumiert 1663 unter der „zemlyá Ilîrskaia“" die Sloweci zadunâiski (hinter der Donau), Bulgaren, Serben und Kroaten. Der erste Autor, der das vorrömische Illyricum schlicht mit Croatia gleichsetzt und ihm nach dem Frieden von Karlowitz 1699 Ragusa, die Hercegovina, die Gebiete nördlich der Save, Slawonien, Istrien, Krain und Serbien zuschlägt, war der genannte Ritter-Vitezović (in dem Traktat Croatia rediviva, 1700). Ebenso setzten Pavle Nenadović und Hristofor Žefarović vierzig Jahre später in der Übersetzung des Wappenbuches von Ritter-Vitezović für den serbischen Metropoliten das alte Illyricum mit dem „Serbsko Carstvo“ der Nemanjiden gleich. Vielleicht kann noch der mit ungeheurer, teils absonderlicher Gelehrsamkeit vorgetragene Entwurf des ragusanischen Jesuiten Ignjat Djurdjević erwähnt werden, der in dem gewaltigen Werk Rerum Illyricarum seine Heimatregion als "Illyricum minus“ beschrieb, umgeben von der großen slawischen Welt mit Polen und Russen, dem „Illyricum magnum“. Das illyrische Ideologem konnte, wie zu sehen ist, räumlich sehr verschiedenen zugeschnitten sein, seine politische Funktion konnte variieren. Einzig von bulgarischer Seite kam früh Widerstand gegen eine Vereinnahmung durch den illyrischen Sog. Nicht ohne Grund erkannte der Erwecker des bulgarischen Nationalbewusstseins, Pajsije Chilendarski, in der illyrischen Konstruktion eine Machenschaft der katholischen Propaganda fide, nicht weniger skeptisch stand man bulgarischerseits dem serbischen, auf den Zaren Stefan Dušan gemünzten Illyricum gegenüber. Für die Bulgaren entfiel somit von vornherein jede illyristische Versuchung.

4 Schmaus 1971, Bd. I, S. 192. 
Auch die Slowenen fungierten lange Zeit am Rande. Das sollte sich in den historischen Wirren zu Anfang des 19. Jahrhunderts grundlegend ändern. Zweimal schufen historische Ereignisse urplötzlich eine politische Verbindung zwischen den kroatischen und den slowenischen Ländern. Beide Prozesse stehen im Zeichen „Illyriens“. Das ist einmal die Schaffung der Illyrischen Provinzen (provinces illyriennes), - mit Westkärnten, Krain, Görz, Triest, Istrien, Fiume, Dalmatien und Teilen Kroatiens - im Frieden von Schönbrunn 1809 als Bestandteil des französischen Kaiserreichs; und zweitens die Gründung des Königreiches Illyrien bald nach dem Sturz Napoleons, 1816, dem außer den slowenischen Ländern zunächst auch Kroatien zugehörte. War auch der politische Sinn beider Kreationen grundverschieden, so gab es gleichwohl eine durchgehende Gemeinsamkeit, nämlich die, dass die beiden illyrischen Staatsgebilde aus kroatischen und slowenischen Ländern gebildet wurden. Aus diesem staatlichen Gerüst wurden erstmals seit dem Aufkommen des illyrischen Ideologems Lösungen denkbar, die außer der politischen auch eine kulturelle, ja sprachliche Annäherung oder gar Vereinigung betreffen konnten. Der slowenische Dichter Valentin Vodnik hat auf beide Illyrien, das Napoleonische wie das habsburgische je mit einem Gedicht reagiert: 1811 schreibt er die Ode Ilirija oživljena, die das von Napoleon wiederbelebte Slowenien (Slowensko) mit Illyrien gleichsetzt, dem aber auch Dubrovnik, Kotor, das Primorje, Oberkrain und das Pokuplje - qua provinces illyriennes - zugehören; 1814 das Gedicht Ilirija zveličena, das in einem Völkerkatalog im Sinne des Austroslawismus die unter Habsburg vereinigten Slawenvölker aufzählt. (Vodnik ist trotz seiner Revokation der Napoleonverehrung schwerwiegender Verfolgung nicht entgangen.) Wir werden sehen, dass es auch aus sprachistorischer Sicht Gründe gab, eine enge sprachliche Verwandtschaft zwischen Slowenen und den sog. „Provinzial- oder Zivil-Kroaten“, d.h. dem kajkavisch sprechenden Teil der Kroaten, anzunehmen. All das zählt mit zu den Voraussetzungen der ,illyristischen Versuchung“.

Das habsburgische Königreich Illyrien bestand von 1816 bis 1849, das heißt, dass die entscheidende Phase der illyristischen Bewegung, 1835-1842 voll in die Zeit des Bestehens dieses politischen Gebildes fällt, woraus sich die anfängliche Duldung, ja Förderung des Illyrismus, als Landespatriotismus, seitens der österreichischen Behörden erklären mag.

Sieht man von Stanko Vraz und wenigen anderen ab, die entsprechende Versuche unternahmen, ließ sich jedoch eine sprachliche und kulturelle Einheit zwischen Kroaten und Slowenen nicht realisieren - aus Gründen, die noch zu nennen sein werden. So blieb der Illyrismus am Ende ein rein kroatisches Projekt, das einen wichtigen Schritt auf dem Wege zu einer Vereinheitlichung der kroatischen Literatursprache und zur Schaffung eines gemeinsamen kulturellen Bewusstseins der Kroaten bedeutet. 


\section{Epistemologische Rekonstruktion}

Begibt man sich an die Rekonstruktion der epistemologischen Schichten oder Episteme, wie sie für die Entstehungszeit des Illyrismus anzusetzen sind, so steht außer Zweifel, dass die „Geistesphysik“ Johann Gottfried Herders entscheidende Impulse auf das illyrische Konzept ausgesendet hat. Bei Tschechen, Slowaken und Südslawen fanden Herders kultur- und geschichtsphilosophischen Thesen lebendigsten Widerhall, da sie Antworten auf Fragen bereithielten, die die samt und sonders in staatlicher Abhängigkeit, sei es im Zarenreich, unter Habsburg oder den Osmanen lebenden - ,schmachtenden“ - Slawen inspirieren mussten. Es sind folgende Herdersche Grundideen, die den Illyrismus - z.T. in der Vermittlung durch Slowaken, Tschechen und Slowenen beflügelten:

1. die Sinngebung der slawischen Geschichte im sog. „Slawenkapitel“ der Ideen zur Philosophie der Geschichte der Menschheit (16. Buch, Kap. IV) ${ }^{5}$;

2. die Ermutigung zum Gebrauch der Muttersprache als dem authentischen Organon zur Erkenntnis der Welt und zur Ausbildung geistigen Lebens dargelegt in Herders Abhandlung über den Ursprung der Sprache

3. die Hervorhebung der Volksdichtung als wichtigster Ausdruck des Nationalgeistes, vorgegeben in den Volksliedern. ${ }^{7}$

Wenn ich hier Herder als den hauptsächlichen Kronzeugen der Slawen herausstelle, bin ich mir bewusst, dass dieser viele zeitgenössische Diskurse aufnahm und auf seine unsystematische Weise aktualisierte und präzisierte. Der tschechische Germanist Vojtěch Jirát hat schon 1929 gezeigt, dass Herder nicht wenige seiner Kenntnisse und Thesen zur Geschichte und zum Volkscharakter der Slawen aus den Göttingischen Gelehrten Anzeigen gewonnen hat. ${ }^{8}$ August Ludwig Schlözer, der Begründer der kritischen Rußlandkunde in Deutschland, postulierte, nicht anders als sein Intimfeind Herder, 1802 (in der Vorrede zu seiner Nestor-Edition):

Die Kultur beginnt zwischen den Völkern nicht eher, als sie in ihren eigenen Sprachen schreiben. ${ }^{9}$

Und auf die Volkspoesie, die Lieder der nördlichen Barden, war man seit Mitte des 18. Jahrhunderts in ganz Europa aufmerksam geworden. Das Bemerkenswerte bei Herder ist, dass die neuen Erkenntnisse und Einsichten von ihm auf neue Art gedeutet und in einen organischen Zusammenhang gebracht wurden, der zu den Fundamenten der romantischen Weltanschauung gehört.

5 Herder 1953, Bd. II, S. 284ff.

6 Ebd., Bd. II, S. 733ff.

7 Ebd., Bd. I, S. 69ff.

8 Jirát 1929.

9 Schlözer 1802. 
Das unterscheidet ihn von der gängigen Aufklärung, nicht zuletzt auch von seinem Antipoden Schlözer. Für diesen ist Geschichte nicht ein organischer Prozess, der aus dem Zusammenwirken oder dem Widerspiel von Völkern, politischen Kräften und virulenten Ideen entsteht, sondern eine Ansammlung von nationalen Teilgeschichten, die er mittels der Linnéschen Klassifikationsmethode in ein vollständiges System überführt. ${ }^{10}$

Märchen, Legenden, die Volksüberlieferung verwirft der kritische Historiker Schlözer als historische Quellen, da sie nicht „wahr“ (historisch wahr) sind. Und den Gebrauch der eigenen Sprache fordert Schlözer aus aufklärerischemanzipatorischen Gründen, nicht als das Organon, das überhaupt kulturelle Identität erst schafft. Obwohl sich mehrere Forscher mit der Bedeutung Herders für die slawischen Wiedergeburtsbewegungen beschäftigt haben ${ }^{11}$, muss gesagt werden, dass das Ausmaß der Herderschen Wirkung erst ansatzweise bekannt ist. Erwähnt wird, dass unter den Slowenen Jernej Kopitar, Urban Jarnik und Janez M. Primič das „Slawenkapitel“ verbreiteten und ausdeuteten, oder dass dieses Kapitel bereits im ersten Jahrgang der Danica Horvatska, Slavonska $i$ Dalmatinska 1835 ins Illyrische übersetzt wurde ${ }^{12}$, nicht aber, dass das gesamte Geschichtsbild der Illyristen auf dem folgenden triadischen Modell beruht:

1. Phase: lichte, idealische Vergangenheit - ein slawisches Goldenes Zeitalter; ${ }^{13}$

2. Phase: Unterjochung und Versklavung der friedfertigen Slawen durch ihre Nachbarn, vor allem Germanen, Magyaren und Tataren;

3. Phase: lichte Zukunft der slawischen Völker. Da diese Vorstellung sehr rasch internalisiert wird, bleibt ihr Urheber ausgespart, wie etwa in Ivan Mažuranićs Programmgedicht Vjekovi Ilirije. ${ }^{14}$

Eduard Winter hat sogar versucht, auch den Austroslawismus Kopitars auf Herder - in der Vermittlung durch Friedrich Schlegel - zurückzuführen, wobei er besonders auf die zivilisatorischen Leistungen und Erfolge der Habsburger Monarchie abhebt, den Slawen europäische Bildung, ,ganz hingeordnet auf Wien und Rom“, zu vermitteln, eine Leistung, die Russland als slawischer Vormacht nicht zuzutrauen sei. ${ }^{15}$ (In der Tat findet sich bei Kopitar Skepsis gegenüber russischen Kulturleistungen; Österreich habe, so schrieb er 1810, die Verpflichtung, die Verbreitung der gelehrten slawischen Sprache und Literatur nicht „,den depravierenden Händen der Russen“ zu überlassen. ${ }^{16}$ )

10 Vgl. Lauer 1985, S. 638ff.

11 Sundhaussen 1973, Barbarić 1978, Ivanišin 1978, Drews 1990.

12 Zu den Quellen der Übersetzung s. Keipert 2000, sowie Pederin 1970.

13 Frangeš 1995, S. 169.

14 Lauer 1978.

15 Winter 1978, S. 207.

16 Pogačnik 1977, S. 108ff. 
Der Austroslawismus, wie er namentlich von Kopitar propagiert wurde, war für die Illyristen ein kulturpolitischer Rahmen, in den das illyristische Konzept eingefügt werden konnte, wenngleich mitunter auch versucht wurde - etwa von Gaj -, die russische Karte zu spielen. Da der Austroslawismus nicht zuletzt auf die Rückführung der orthodoxen Slawen unter das Szepter Roms abzielte, löste er bei den Serben und noch mehr bei den Bulgaren die schon erwähnten Reflexe aus, was sich mittelbar auf die Inakzeptanz des Illyrismus bei diesen Nationen auswirkte.

Auf eine andere Weise lieferte Kopitars Austroslawismus Gründe, die ein Zusammengehen von Slowenen und Kroaten suggerierten. Gemeint ist Kopitars These von der pannonischen Herkunft des altslawischen Schrifttums, die den Slowenen eine Schlüsselrolle bei der Entstehung der slawischen Kultur zuschrieb. Die mit dieser These postulierte Einheit von slowenischer (windischer) und provinzialkroatischer (kajkawischer) Sprache aber lief den Bestrebungen der Illyristen, die das Kajkawische ja gerade in seiner literatursprachlichen Relevanz eliminieren wollten, diametral entgegen.

Von zeitgenössischen Autoren wurde bereits auch versucht, sehr wahrscheinlich mit dem Ziel, die politische Brisanz des Illyrismus zu mindern, eine Unterscheidung zwischen Illyrismus (ilirizam) und Kroatismus (kroatizam) vorzunehmen. Dahinter verbirgt sich nicht nur eine semantische Differenzierung, sondern zugleich auch die Maßgabe, behördliche Zweifel an der politischen Unschuld des Illyrismus zu entkräften. Autor des „,berühmten Feuilletons“ (znamenitoga feljtona) zu diesem Thema aus dem Jahre 1842 ist Ljudevit Vukotinović. Er versichert gleich zu Anfang, dass der Illyrismus im politischen Sinne nichts bedeute ${ }^{17}$, während der Kroatismus ,unser politisches Leben“ bezeichne. ${ }^{18}$ Im königlich-ungarischen Sabor seien alle nur Kroaten, doch ansonsten gehöre man dem gleichen großen Stamme an wie Bosniaken, Grenzer, Herzegowiner, Serben usw. Vukotinović vergleicht das mit den deutschen Verhältnissen: Sachsen und Württemberger seien nur im konstitutionellen Sinne Sachsen und Württemberger, im genealogischen aber seien sie Deutsche. ${ }^{19}$ Der Kampf der Provinzial-Kroaten, d.h. der Kajkawen, um ihre konstitutionellen Rechte, wird nicht in Frage gestellt, aber er darf nicht mit dem kulturpolitischen Zielen der Illyristen verwechselt werden. Und hier nennt Vukotinović die entscheidende Devise; sie lautet: „Der literarische Illyrismus ist unser Seelenleben“" (Ilirizam slovnički je život naš duševni). ${ }^{20}$ Und dafür benötige man eine schöne, ausbildungsfähige Sprache (lijep i izobraženosti shodan jezik) und eine große Lesergemeinde (veliko čitajuće općinstvo). ${ }^{21}$ Das

17 Ravlić 1965, Bd. II, S. 66.

18 Ebd., S. 67.

19 Ebd.

20 Ebd.

21 Ebd., S. 69ff. 
sprachpolitische und das kommunikationspolitische Argument spielt im illyristischen Diskurs eine entscheidende Rolle.

\section{Die sprachliche Situation}

Die sprachliche Situation war in den slowenischen wie in den kroatischen Ländern um das Jahr 1830 ungeklärt und offen. Zwar gab es in beiden Bereichen alte Traditionen schriftlicher Literatur, die bis ins Mittelalter zurückreichten. Die slowenische Sprache hatte in der Reformationszeit eine nachhaltige Fixierung erhalten, die Bohoričica; die kroatische blickte in verschiedenen Regionen, vor allem aber in Dubrovnik, auf einzigartige literarische Leistungen im 16. und 17. Jahrhundert zurück. Doch waren diese Traditionen in stark voneinander abweichenden Dialektsträngen realisiert - bei den Kroaten: čakawisch, kajkawisch und štokawisch in zwei Varianten, ragusanisch und slawonisch; bei den Slowenen: krainisch und südoststeirisch. ${ }^{22}$ In beiden Sprachbezirken konkurrierten überdies verschiedene orthographische Systeme miteinander. Im sogenannten slowenischen ABC-Krieg traten 1833 wenigstens drei unterschiedliche Konzepte auf den Plan; rechnet man die magyarische Schreibweise im Übermurgebiet (Prekomurje) hinzu, so sind es vier. Auch bei den Kroaten stehen wenigstens vier Alternativen zur Disposition: kajkawisch, slawonisch, ragusanisch, čakawisch, alle bedrängt durch die von Gaj bevorzugte tschechische Lösung mit diakritischen Zeichen.

Unter Berufung auf das Herdersche Muttersprachkriterium hätte jede gegebene Dialektvariante literaturtauglich sein können oder müssen, was freilich zu einer Atomisierung der Sprachschichtung hätte führen müssen. Und so zeichnen sich sehr schnell Lösungen ab, die einmal auf größere, einheitliche Sprachblöcke abzielen, dann aber auch die kulturologischen Zwecke der Sprache in Rechnung stellen. Im kroatischen und slowenischen Umkreis lassen sich einige der grundlegenden Entscheidungen parallel setzen:

1. Der Verzicht auf das Kajkawische, damals von ca. 200.000 Sprechern gesprochen, zugunsten der von 4-5 Mio. gesprochenen Štokaviština bei Kroaten und Serben; und der Verzicht auf das Südoststeirische zugunsten des Krajnischen bei den Slowenen. Das Ziel, einen möglichst großen literarischen Kommunikationsraum zu schaffen, spielt in beiden Fällen die entscheidende Rolle.

2. Das Verwerfen der von Kopitar und Metelko (metelčica) geförderten und geforderten Sprache, die zwar dem Herderschen Postulat folgte, aber vor allem auf die Schriftbedürfnisse der Landbevölkerung zugeschnitten war. Diese Sprache entsprach den kulturellen Anforderungen eines städtisch-

22 Slodnjak 1958, S. $119 \mathrm{ff}$. 
bürgerlichen, literarisch interessierten Publikums ebenso wenig wie das klerikal-aufklärerische kajkawische Schrifttum bei den Provinzial-Kroaten.

In beiden Bereichen entsteht also das Gerüst der Literatursprache unter deutlicher, bewusster Abweichung vom Herderschen Muttersprachkriterium. Hier ist anzumerken, dass zwischen den südoststeirischen Dialekten des Slowenischen und dem kroatischen Kajkawisch - damals noch im engeren Sinne die kroatische (chorvatische) Sprache! - eine größere Affinität bestand, die möglicherweise den Grund für eine ganz andere Option hätte abgeben können. (Schlözer hatte noch 1771 in seiner Klassifikation der slawischen Sprachen gefragt, ob nicht das Kajkawische (Chorvatische) eine Varietät des Windischen (Slowenischen) darstelle - oder umgekehrt. ${ }^{23}$ )

3. Bei Slowenen und Kroaten fungierte die deutsche Sprache und die reichen Hervorbringungen der damaligen deutschen Literatur als ein unabdingbares Vorbild, das zu erreichen war. Fast alle bedeutenden südslawischen Autoren jener Jahre haben deutsch geschrieben und gedichtet. ${ }^{24}$ Nicht selten gewinnt man den Eindruck, es sei ihnen in der hochelaborierten deutschen Dichtsprache wesentlich leichter gefallen, Verse zu schreiben, als in dem noch schwerfälligen eigenen Idiom. Die deutschen Gedichte Prešerens etwa fügen sich nahtlos in den klassisch-romantischen Kanon der deutschen Literatur ein. Bemerkenswert auch, dass die slowenischen und kroatischen Literaten ihren Briefwechsel zum größten Teil auf Deutsch führten.

\section{Die Optionen von Prešeren und Vraz}

Die hier knapp skizzierten Modellvorstellungen zum illyrischen Ideologem, zu den geistesgeschichtlichen Epistemen und zur Sprachsituation um 1830 zeigen eine hochgradige Widersprüchlichkeit, ja Beliebigkeit, die die Schriftsteller und Dichter der Zeit in ein grausiges Dilemma stürzte. Das illyrische Konzept konnte nach Umfang und Ziel beliebig ausgefaltet werden. Slowenen und Bulgaren konnten darin enthalten sein - oder auch nicht. Ihm widersprach im ganzen Kopitars slowenozentrischer Karantano-Pannonismus, der Karantanien (Kärnten) als die Urheimat der Slawen annahm und die Entstehung des Altkirchenslawischen nach Pannonien verwies. ${ }^{25}$ Herders arbiträrer Hinweis auf die Muttersprache als Quelle der Kultursprache konnte enger oder weiter interpretiert werden - obwohl Herder eigentlich keinen Zweifel daran lässt, was er meint: Er beschreibt ganz konkret das Bild, wie das Kind, auf dem Schoß er Mutter sitzend, erste Laute und Worte erlernt. ${ }^{26}$ War nun, lautet die Kardinal-

23 Schlözer 1771, S. 334.

24 Vgl. den Beitrag von Peter Scherber im vorliegenden Band.

25 Pogačnik 1977, S. 105ff.

26 Herder 1953, Bd. I, S. 809. 
frage, jeder lokale Dialekt oder Subdialekt literatursprachfähig - also etwa das Südoststeirische bei den Slowenen -, oder waren es nur die ,zentralen“ Idiome - wie das Krainische der Čop-Prešerenschen Option; oder musste Sprache gar für größere Bevölkerungskonglomerate erst künstlich gebildet werden wie die ,illyrische“ Sprache der Illyristen? Da auch die Isoglossen widersprüchlich gedeutet wurden und zudem bei Slowenen wie Kroaten - wie erwähnt - eine reiche Palette an orthographischen Systemen bereitstand - Bohoričica, Dajnica, Metelčica, Gajica des alten und neuen Typus, magyarische Orthographie und nicht zu vergessen Vuk Karadžićs fonetski pravopis - eröffneten sich im genannten Zeitraum zahllose, äußerst verwirrende Möglichkeiten und Solutionsofferten, die von den südslawischen Autoren aufgegriffen oder verworfen werden konnten.

Hier zeigt sich nun, dass von unseren beiden slowenischen Dichtern, beide mit besonderem literarischem Instinkt begabt, völlig unterschiedliche Lösungen ergriffen wurden, die vom Grundsatz her verschieden waren, wenn auch von den jeweiligen Argumenten her verständlich.

Prešeren, unterstützt von Matija Čop, dem slowenischen Arbiter elegantiarum et litterarum, sowie dem Kreis um den Almanach Krajnska čelica, vermag sich, mit schlafwandlerischer Sicherheit, nur auf eines einzulassen: zu dichten auf der künstlerischen Höhe Petrarcas oder der deutschen klassischromantischen Poesie im eigenen, krainischen, durch die Jahrhunderte vorbereiteten und jetzt aktualisierten sprachlichen Medium. So verwirft er die slowenischen Subdialekte (Vraz) oder die erbauliche Rustikalsprache (Metelko, Kopitar) ebenso wie die südslawische Kunstsprache der Illyristen. Solche literarische Tat konnte allein dem dichterischen Genius gelingen. Da alle literatursoziologischen Voraussetzungen für die Etablierung großer Literatur bei der kaum eine Million Sprecher zählenden Nation fehlten, war das Scheitern des Prešerenschen Unterfangens keineswegs von vornherein auszuschließen. Letztlich hat ihn der weltliterarische Rang seiner Poesie gerettet und gerechtfertigt.

Ganz anders Stanko Vraz. Ihm blieben gleich zu Beginn seiner literarischen Laufbahn zwei bittere Erfahrungen nicht erspart. Einmal musste er erleben, dass das wichtigste Organ der slowenischen Literatur jener Jahre, der Almanach Krajnska cbelica, vor allem aus wirtschaftlichen Gründen nach dem 4. Band sein Erscheinen einstellte (ein letzter Band erschien 1848). Die Texte, die er eingereicht hatte, wurden nicht gedruckt. Die andere Erfahrung zeigte ihm, dass den Krainer Literaten sein südoststeirischer Dialekt nicht genehm war. Die Čop-Prešerensche Richtung ließ eine Absenkung des literatursprachlichen Niveaus auf das eines rustikalen Subdialektes nicht mehr zu. Vraz' Option für die illyristische Sache - sein Erliegen der illyristischen Versuchung - mag sich zunächst auf die Nähe seines südoststeirischen Dialekts zum Kajkawischen gegründet haben; nachdem das Kajkawische jedoch von den Illyristen zugunsten des Štokawischen aufgegeben worden war, traten zwei andere gewichtige Faktoren hinzu: die illyristische Begeisterung, die in Ljubljana kein Pendant besaß, und der ungleich größere Rezeptionsraum - für einen Literaten, der 
von der Literatur leben wollte und musste, buchstäblich eine Überlebensfrage, über die sich Vraz genauestens Rechenschaft gab. Da er mit seinen Werken in illyrischer Sprache durchaus reussierte und sich mit ihnen das Ansehen des, neben Ivan Mažuranić und Petar Preradović, besten illyristischen Dichters erwarb, ohne sich von der slowenischen Literatur vollständig zu lösen, fällt es nicht schwer, Verständnis für seine Option aufzubringen, mag Vraz aus slowenischer Sicht mitunter auch als Abtrünniger, ja Verräter am Slowenentum erscheinen.

Dass gerade die besten Autoren, Prešeren und Vraz, die vor ihnen aufgetürmten Widersprüche nicht nur als modellartige Virtualität, sondern als existentielle Entscheidungssituationen erlebten, möchte ich am Schluss anhand einiger Briefäußerungen aus dem auf deutsch geführten Briefwechsel der beiden Dichter illustrieren, um damit zugleich die ,illyristische Versuchung“ als historisch-konkreten Vorgang darzubieten.

Als der in Ljubljana lebende Pole Emil Korytko 1838 mit Unterstützung Prešerens daran ging, eine Sammlung slowenischer Volkslieder herauszugeben, wandte er sich auch an Ljudevit Gaj in Zagreb, um den Band in dessen Druckerei setzen zu lassen. Gaj erklärte seine Bereitschaft, den Band zu drucken, forderte aber die Anpassung der Ausgabe an die illyristischen Normen. Prešeren fühlte sich herausgefordert und antwortete Gaj in einem an Vraz adressierten Schreiben vom 19. Juli 1838, in dem er den Gedanken, das Slowenische als Literatursprache aufzugeben und das „Serbische“ zur höheren Literatursprache zu machen, mit Entschiedenheit zurückwies. ${ }^{27}$ (Die Volksliedsammlung erschien später unter dem Titel Illirske pesmi krajnskega naroda, gedruckt in der illyristischen Gajica. ${ }^{28}$ )

Schon zuvor hatte Prešeren in einem Brief vom 5. Juli 1837 mit großer Klarheit das einzige, genuine Ziel des slowenischen Schriftstellers definiert:

Die Tendenz unserer Carmina und sonstigen literärischen Thätigkeit ist keine andere als unsere Muttersprache zu kultivieren; habt Ihr ein anderes Ziel, so werdet Ihr es schwerlich erreichen. Die Vereinigung aller Slawen zu e i n e r Schriftsprache wird wahrscheinlich ein frommer Wunsch bleiben. Es dürfte Euch nicht so leicht gelingen den steirisch kroatischen Dialekt auf den philologischen Autokraten Thron zu erheben. Unterdessen auch verkehrtes Streben ist besser als Apathie gegen alles Vaterländische. ${ }^{29}$

Vraz hingegen verteidigte seine Abkehr von der slowenischen Literatur in seinem Brief an Prešeren vom 19. November 1837:

Ich habe auf Deinen Beitritt zu meinen Ansichten gerechnet, und darauf bauend noch das Vegetieren der slowenischen Literatur für möglich gehalten. Da aber das nicht erfolgt ist, so habe ich mich seit verfloss. Frühjahre vom undankbaren Felde,

27 Nach Paternu 1993, S. 244.

28 Ebd.

29 Prešeren 1964, S. 342. 
das ich fünf Jahre mit aller Liebe bebaute, zurückgezogen und mich den begeisterten jungen Illyriern angeschlossen und denke nicht wieder zurückzutreten. Mit Slowenien hab' ich es abgetan, zumal da ich auf meiner letzten Reise alle meine Schriften, die ich von dem Jahre 1832-36 in slow. Sprache besaß, verlor. Seit dem Jahre schreibe ich nur illyrisch. Was Herr Kastelic von mir besitzt, sind deshalb als Opera posthuma zu betrachten. ${ }^{30}$

Vraz hat dabei immer im Sinn, was er im Brief vom 1. August 1837 als ,die numerische Inferiorität unseres slowenischen Völkleins“ bezeichnet. Sie mache es notwendig, sich an irgendeinen benachbarten und verwandten Volksstamm anzuschließen. Während Prešeren mit ironischer Überlegenheit seine Position vertrat, legte Vraz nun, im Brief vom 25. Dezember 1840, in aller Offenheit, mit Zahlen argumentierend, seine literatursoziologischen Gründe dar:

Sage mir, wie viel gibt es unter der Million Slowenen Abnehmer für ein wissenschaftliches oder belletristisches Werk in dem Hausdialekte? und wie viele kann es bei der sanguinischen [temperamentvollen] Hoffnung in der Zukunft geben? Nie soviel, dass die Druckkosten bestritten werden können, um wie viel weniger soviel, dass ein slow. Schriftsteller von dem Ertrage seiner Werke honett leben könnte. So lange die Literaten einer Sprache nicht wenigstens diese Hoffnung hegen dürfen, gibt es keine Literatur. Die Kultur einer jeden Sprache, die etwas in der Welt bedeuten will, muss sich wenigstens auf eine Volksmasse von 3-4 Millionen stützen. ${ }^{31}$

Obwohl Vraz damit recht hatte und Prešeren nicht zuletzt der Leidtragende der geschilderten Verhältnisse war, schmähte dieser Vraz mit dem folgenden Distichenepigramm:

Od drugih manjši in častèn manj rod je slovenski, lákota sláve, blagá vleče pisárja drugám ... ${ }^{32}$

[Geringer als andre und arm an Ehren ist der slowenische Stamm, Hunger nach Ruhm, nach Wohlstand lockt den Scribenten hinweg...]

Wir sehen: Letztlich argumentierten beide Dichter in der damaligen Situation mit durchaus nachvollziehbaren Gründen. Aus heutiger Sicht nimmt sich freilich alles ganz anders aus. Vraz hat, sich der slowenischen Literatur versagend, große Verdienste um die Entwicklung der kroatischen Literatur erworben. Er ist ein kroatischer Dichter geworden - mit einigen slowenischen Beimengungen. Prešeren aber hat mit seiner donquichotischen Entscheidung nicht nur die slowenische Literatur auf eine unerhörte Höhe gehoben, sondern eben dadurch bewirkt, dass sich das literarische Leben in seinem kleinen Lande in einer Weise entfaltete, dass Slowenien heute wohl eines der Länder ist, die ein ganz erstaunlich dichtes literarisches Leben vorzuweisen haben: mit einer besonders großen Zahl an Schriftstellern, Kritikern und Literaturwissenschaftlern,

30 Paternu 1993, S. 246.

31 Ebd., S. 248ff.

32 Prešeren 1964, Bd. I, S. 93. 
einer reichen literarischen Produktion, unbändigen Übersetzungsaktivitäten, einem breit entwickelten Lesepublikum und einem wohlorganisierten Literaturmarkt. Prešeren hat dieses blühende Literaturleben wohl geahnt, aber leider nicht mehr erlebt. Vraz hingegen war zu ungeduldig und zu skeptisch, um es überhaupt für möglich zu halten. Darum vor allem ist er der ,illyristischen Versuchung“" erlegen.

\section{Literaturverzeichnis}

Barac, Antun, 1964: Hrvatska književnost. Knjiga I. Književnost ilirizma. Zagreb.

Barbarič, Štefan, 1978: „Herder in der slowenischen Literatur“, in: Ziegengeist, Gerhard - u.a. (Hrsg.): Johann Gottfried Herder. Zur Herder-Rezeption in Ost- und Südosteuropa. Berlin, 117-124.

Danica Ilirska. Reprint. Zagreb 1970.

Drechsler, Branko, 1909: Stanko Vraz. Studija. Zagreb.

Drews, Peter, 1990: Herder und die Slawen. Materialien zur Wirkungsgeschichte bis zur Mitte des 19. Jahrhunderts. München.

Frangeš, Ivo, 1995: Geschichte der kroatischen Literatur. Von den Anfängen bis zur Gegenwart. Köln. Weimar. Wien.

Herder, Johann Gottfried, 1953: Werke in zwei Bänden. München.

Ivanišin, Nikola, 1978: „Herder und der Illyrismus“, in: Ziegengeist, Gerhard - u.a. (Hrsg.): Johann Gottfried Herder. Zur Herder-Rezeption in Ost- und Südosteuropa. Berlin, 125-131.

Ježić, Slavko (Hrsg.), 1934: Ilirska antologija. Književni dokumenti hrvatskog preporoda. Zagreb.

Jirát, Vojtěch, 1929: „Slavisches in den ,Göttingischen Gelehrten Anzeigen“ 17391790“, in: Xenia Pragensia ... Prag, 121-181.

Keipert, Helmut, 2000: „Herders ,Slawen-Kapitel` in der ,Danica Horvatska“, Slavonska i Dalmatinska (1835)“, in: Jekutsch, Ulrike / Kroll, Walter (Hrsg.): Slavische Literaturen im Dialog. Festschrift für Reinhard Lauer zum 65. Geburtstag. Wiesbaden, 129-140.

Kolo 1842.-1853. I-IV. Reprint. Zagreb 1993.

Lauer, Reinhard, 1974: „Genese und Funktion des illyrischen Ideologems in den südslavischen Literaturen (16. bis Anfang des 19. Jahrhunderts)“, in: Grothusen, Klaus-Detlev (Hrsg.): Ethnogenese und Staatsbildung in Südosteuropa. Göttingen, 116-143.

Lauer, Reinhard, 1978: „Ivan Mažuranićs ,Vjekovi Ilirije“ - Strukturen und Sinnbezüge“, in: Holthusen, J. / Kasack, W. / Olesch, R. (Hrsg.): Slawistische Studien zum VIII. Internationalen Slavistenkongress in Zagreb. Köln-Wien, 299-321.

Lauer, Reinhard, 1985: „Schlözer und die Grundlegung slavistischer Methodologie“, in: Zeitschrift für Slawistik 30 (1985), 5, 634-644.

Paternu, Boris, 1993: France Prešeren. Ein slowenischer Dichter 1800-1849. (Aus dem Slowenischen von Klaus Detlef Olof). München. 
Pederin, Ivan, 1970: „Rodoljubni dodaci i ispuštanja u prijevodu Herderova poglavlja ,Slavenski narodi“, koji je objelodanjen u ,Danici Ilirskoj‘1835. godine“, in: Radovi Filozofskog Fakulteta u Zadru. Razdio lingvističko-filološki (5). 8 (1970), 264-270.

Petré, Fran, 1939: Poizkus ilirizma pri slovencev (1835-1849). Ljubljana.

Pogačnik, Jože, 1977: Von der Dekoration zur Narration. Zur Entstehungsgeschichte der slowenischen Literatur. München.

Pogačnik, Jože, 1978: Bartholomäus Kopitar. Leben und Werk. München.

Pogačnik, Jože, 1998: Slovenska književnost. I. Ljubljana.

Prešeren, France, 1964: Poezije in pisma. Uredil Anton Slodnjak. Ljubljana.

Ravlić, Jakša (Hrsg.), 1965: Hrvatski narodni preporod. I-II. Zagreb.

Schlözer, August Ludwig, 1771: Allgemeine Nordische Geschichte ... Halle.

Schlözer, August Ludwig, 1802-1809: Nestor. Russische Annalen in ihrer slavonischen Grundsprache. Göttingen.

Schmaus, Alois, 1971: Vincentius Pribojević - ein Vorläufer des Panslavismus (1953); Gesammelte slawistische und balkanologische Abhandlungen, Bd. I. München.

Slodnjak, Anton, 1958: Geschichte der slowenischen Literatur. Berlin.

Sundhaussen, Holm, 1973: Der Einfluss der Herderschen Ideen auf die Nationsbildung bei den Völkern der Habsburger Monarchie. München.

Vraz, Stanko, 1953-1955: Pjesnička djela I-III. (Hrsg. Ježić, Slavko) Zagreb.

Vraz, Stanko / Preradović, Petar, 1954: Djela. Zagreb.

Winter, Eduard, 1978: Herder und die Entwicklung eines wirklichen Austroslawismus über Friedrich Schlegel, in: Ziegengeist, Gerhard u.a. (Hrsg.): Johann Gottfried Herder. Zur Herder-Rezeption in Ost- und Südosteuropa. Berlin, 205-211.

Ziegengeist, Gerhard u.a. (Hrsg.), 1978: Johann Gottfried Herder. Zur Herder-Rezeption in Ost- und Südosteuropa. Berlin. 